\title{
Study on the Electrochromism of the Complexes of Viologens and their Acceptors
}

\author{
Liu Changyan, Ii Guifan, Yang Yukun, and Li Yongfeng \\ Institute of Chemistry, Academia Sinica, Beijing 100080 \\ China
}

\begin{abstract}
The electrochemical behaviour of viologens and their complexes with acceptors was investigated. We found that there are three redox states for viologens, and four states for the complexes which depended on the concentration and the affinity of acceptor. Generally the number of redox states is only two. Additional redox states result from molecular interaction, exactly from the charge transfer interaction between molecules in the system. The affinity of the compound increased as a result of complexing with an acceptor. Particularly, the complexation of radical salt and acceptor was discussed in detail.
\end{abstract}

\section{Introduction}

The electrochemistry of viologens has been widely investigated $[1,2,3]$. The electrochemical behaviour of the complexes of viologens and some acceptors has been studied in 
this paper. We found that the effect of the molecular interaction on the electrochromic property of material must be considered in electrochemical system.

Electrochemical redox processes of 4,4'-bipyridinium salt are expressed as follows.
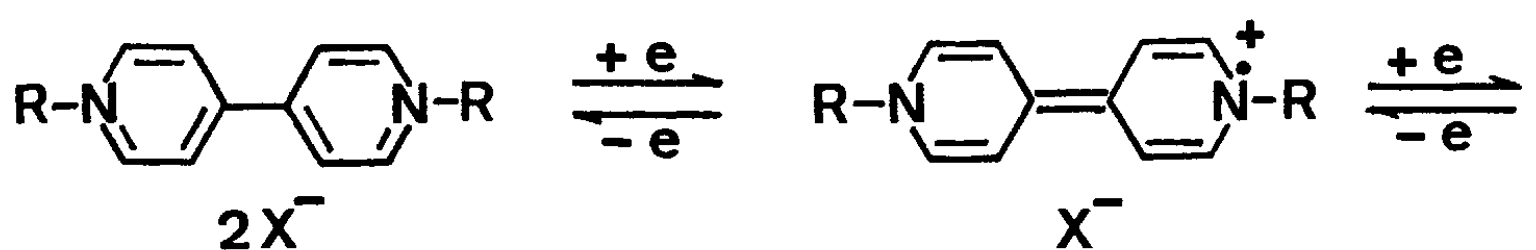

$2 x^{-}$
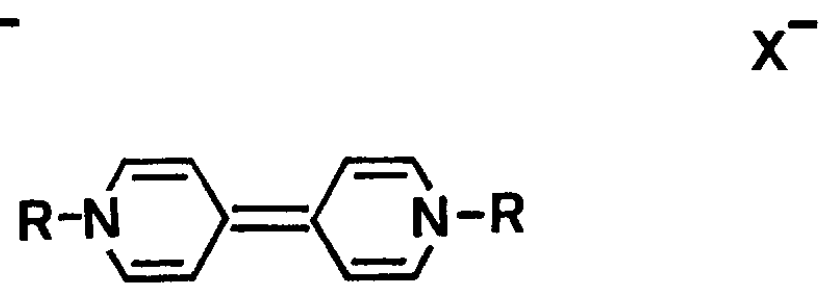

First, we performed a simple experiment in order to extend above viewpoint. When the colourless solution of viologen with $\mathrm{R}=p-\mathrm{CNC}_{6} \mathrm{H}_{4}$ substituent is mixed a small quantity of sodium hyposulfite, a green colour was appeared. The radical ion salt can be obtained by means of reducing viologen. The green solution was decolourized rapidly, and transformed into yellow when TCNQ was poured into the solution. But it reappeared green colour by mixing some reductant again.

The radical ion salt and the compound described above are good donors, and able to form charge transfer complexes with some acceptors. The bipyridinium salt also plays as an acceptor in the system. The reducing potential generally corresponds to its affinity[4]. When the affinity of complexating is different from uncomplexating, the difference can be determined certainly.

\section{Experimental}

$4,4^{\prime}$-Bipyridium bromates $\left(\mathrm{R}=\mathrm{CH}_{3}, \mathrm{CH}_{3} \mathrm{CH}_{2} \mathrm{CH}_{2}, \mathrm{PhCH}_{2}\right.$, and $p-$ $\mathrm{CNC}_{6} \mathrm{H}_{4}$ ) were synthesized and purified. 7,7,8,8-Tetracyano- 
quinodimethane(TCNQ) and chloranil(CA) were obtained from Aldrich Chemical Company, Inc. The cyclic voltammograms were recorded with an EG\&G 370 electrochemical system. Lithium perchlorate dissolved in acetonitril was used as support elctrolyte. During experiment the oxygen in the solution was removed by the passage of argon.

\section{Results and Discussion}

Cyclic voltammetry on platinum-wire working electrode was employed for the determination of reducing potentials (Table).

Table: Results for cyclic voltammetry on platinum-wire working electrodes.

\begin{tabular}{cccccc}
\hline $\begin{array}{c}\text { Viologen } \\
\text { R }\end{array}$ & Acceptor & & Cathode $(\mathrm{mV})$ & \\
\hline Methyl & & $-\mathrm{E}_{1}$ & $-\mathrm{E}_{2}$ & $-\mathrm{E}_{3}$ & $-\mathrm{E}_{4}$ \\
Methyl & TCNQ & 450 & 640 & 760 & 940 \\
& TCNQ & -320 & 30 & & \\
Methyl & CA & 290 & & 700 & 925 \\
& CA & -220 & -90 & & \\
Propyl & & 280 & & 690 & 900 \\
Propyl & TCNQ & 290 & & 760 & 920 \\
Propyl & CA & 290 & & 730 & 900 \\
Benzyl & & 230 & & 640 & 820 \\
Benzyl & TCNQ & 250 & 540 & 640 & 820 \\
Benzyl & CA & 230 & & 640 & 820 \\
\hline
\end{tabular}

The typical redox curves in negative potential region are shown as follows. 


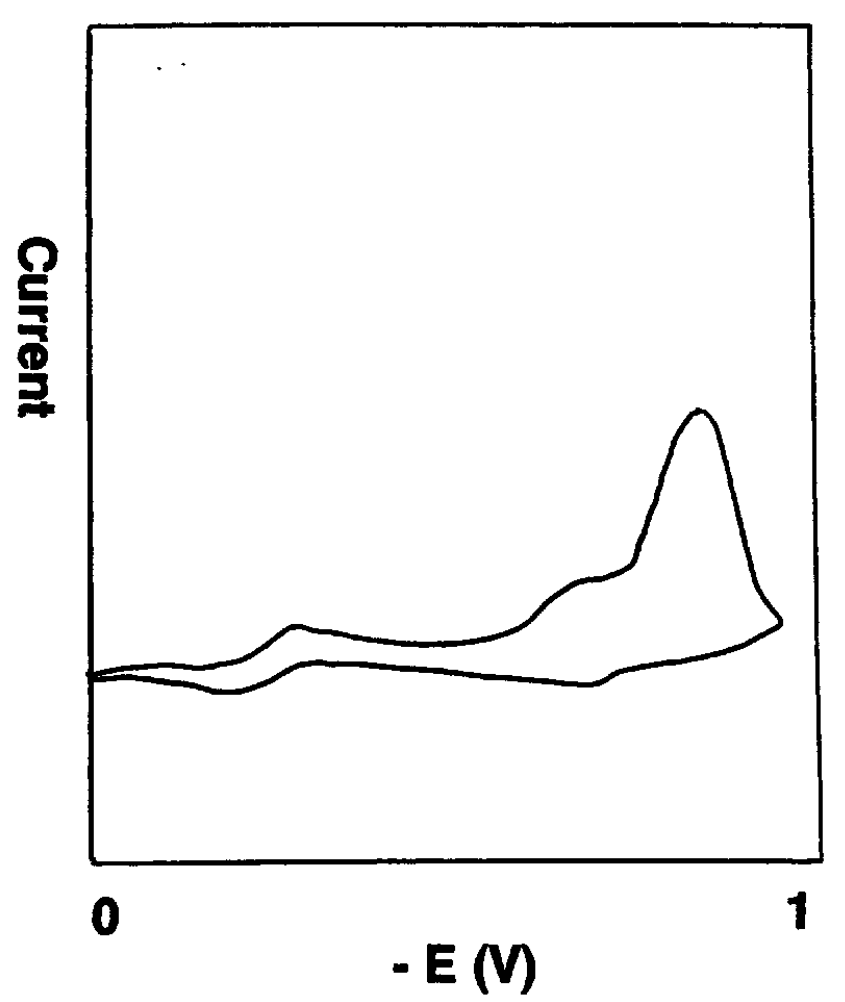

[ a ]

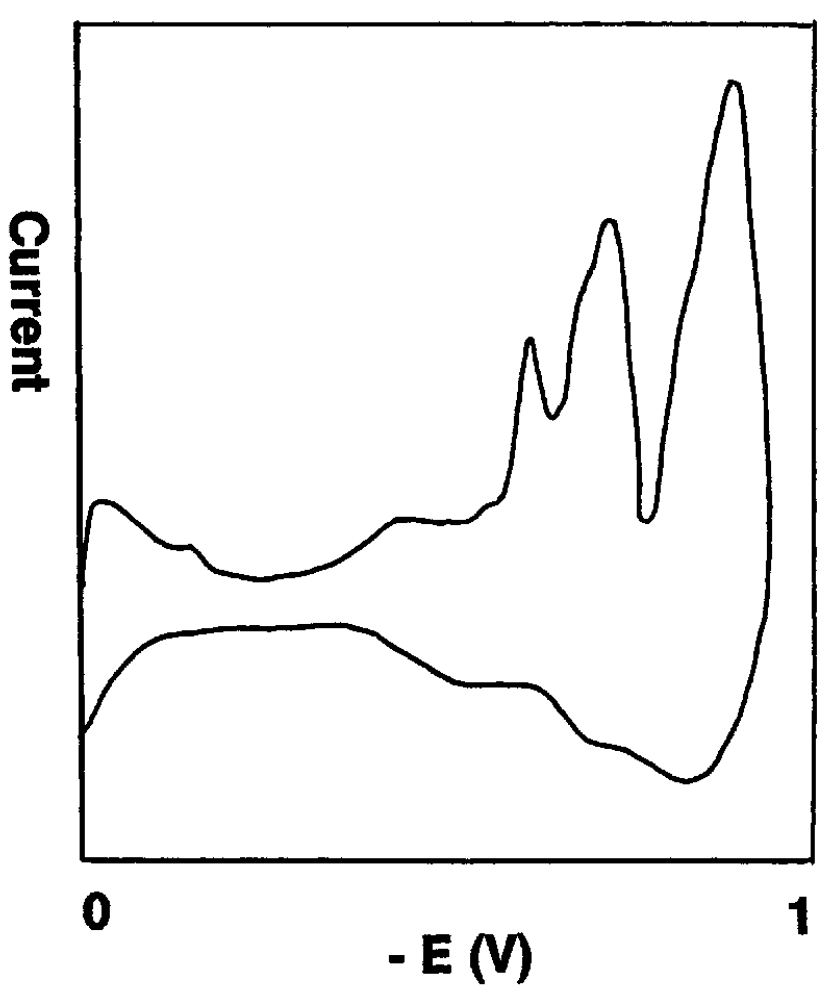

[ $b$ ]

Fig: Cyclic voltammograms,

[a]: for bipyridium salt, [b]: for complex.

It was observed that all of viologens were possessed of three redox potentials as shown in Fig. [a]. Obviously, $E_{1}$ corresponds to the first reaction step for viologen. There are two potentials for the second, symbolizing as $E_{3}$ and $E_{4}$ for convenience. We consider that the phenomenon is attributed to molecular interaction, not to experimental errors.

When TCNQ and CA were mixed in the solution of viologen, we obtained interesting results. The molecular interaction in the system is verified further. The redox potentials are positive or near zero (cf. Table). When the addition of TCNQ approached to the quantity of viologen, four redox states appeared in the system (except for TCNQ) as shown in Table and 
Fig. [b]. $E_{2}$ expresses a new reducing state corresponding to the complexating of radical ion and acceptor.

In the case that the radical ion is oxidized slightly by the acceptor, the distribution of electron cloud on the molecular skeleton would change as follows. The energy level

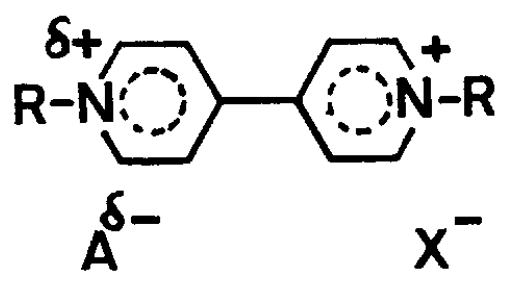

changes also, so that the affinity increases. The appearance of the new peak arised from the complexation depends on the concentration of complexating radical ion, and the reducing potential depends on charge transfer degree. The concentration increases with the increase of acceptor in the system. The degree of charge transfer depends on the strength of donor and acceptor. When CA or a small quantity of TCNQ is mixed in the solution, a new peak (for example, $E_{2}$ ) can not be observed, although the current in $\mathrm{E}_{3}$ increase under the influence of them with increasing the concentration of them. Now we can explain the colour change in the first performed experiment for the reason given above.

Barltrop and his colleagues [2] found that insoluble radical ion salt was deposited as film on the cathode, and the absorption band of the radical film was seen to have developed a shoulder. It falls within same reason.

The reducing potential $E_{1}$ of the complex of methylviologen and TCNQ is higher than that of viologen (cf. Table). It is perhaps is influenced by multiple peaks.

\section{Conclusion}

Molecular interaction produces the effects on the redox 
potential of viologen. It is of importance to the utilization of viologen as display. A multiple colour display will be achieved easily.

Acknowledgment: This work was supported by Prof. Yan Baozhen and Miss Yang Jing.

\section{References}

1. N. J. Goddard, A. C. Jackson, and M. G. Thomas: J. Electroanal. Chem. Interfacial Electrochem., 159,(1983), 325.

2. J. A. Barltrop and A. C. Jackson: J. Chem. Soc., Perkin Trans., 2, (1984), 367.

3. Masao Yamana and Tsutomu Kawata: Nippon Kagaku Kaishi: (1977), 941.

4. T. K. Mukherjiel: Tetrahedron, 24, (1968), 721. 